\title{
Stability and Relaxation Mechanisms of Citric Acid Coated Magnetite Nanoparticles for Magnetic Hyperthermia
}

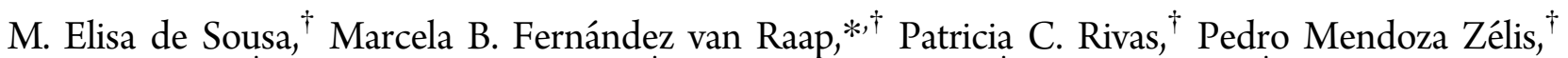 \\ Pablo Girardin, ${ }^{\dagger}$ Gustavo A. Pasquevich, ${ }^{\dagger}$ Jose L. Alessandrini, ${ }^{\dagger}$ Diego Muraca, ${ }^{\dagger}$ \\ and Francisco H. Sánchez ${ }^{\dagger}$ \\ ${ }^{\dagger}$ Instituto de Física de La Plata (IFLP- CONICET), Departamento de Física, Facultad de Ciencias Exactas, Universidad Nacional de \\ La Plata (UNLP), c.c. 67, 1900 La Plata, Argentina \\ "Instituto de Física "Gleb Wataghin" (IFGW), Universidade Estadual de Campinas, Brazil
}

Supporting Information

ABSTRACT: Magnetite $\left(\mathrm{Fe}_{3} \mathrm{O}_{4}\right)$ nanoparticles are proper materials for Magnetic Fluid Hyperthermia applications whenever these conjugate stability at physiological (neutral $\mathrm{pH}$ ) medium and high specific dissipation power. Here, magnetite nanoparticles 9-12 nm in size, electrostatically stabilized by citric acid coating, with hydrodynamic sizes in the range $17-30 \mathrm{~nm}$, and well dispersed in aqueous solution were prepared using a chemical route. The influence of media acidity during the adsorption of citric acid (CA) on the suspension's long-term stability was systematically

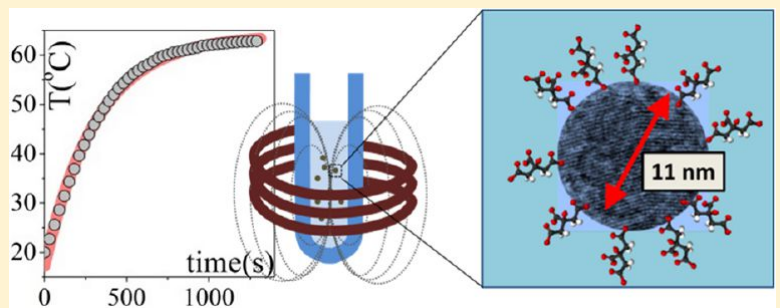
investigated. The highest content of nanoparticles in a stable suspension at neutral $\mathrm{pH}$ is obtained for coating performed at $\mathrm{pH}=4.58$, corresponding to the larger amount of CA molecules adsorbed by one carboxylate link. Specific absorption rates (SARs) of various magnetite colloids, determined calorimetrically at a radio frequency field of $265 \mathrm{kHz}$ and field amplitude of $40.1 \mathrm{kA} / \mathrm{m}$, are analyzed in terms of structural and magnetic colloid properties. Larger dipolar interactions lead to larger Néel relaxation times, in some cases larger than Brown relaxation times, which in the present case enhanced magnetic radio frequency heating. The improvement of suspension stability results in a decrease of SAR values, and this decrease is even large in comparison with uncoated magnetite nanoparticles. This fact is related to interactions between particles.

\section{INTRODUCTION}

Stable dispersion of magnetic nanoparticles (MNPs) has attracted much attention, in a first stage because of their rheological properties ${ }^{1}$ that allow dynamic control by applied fields and more recently due to their widespread biomedical diagnostic and therapeutic applications ${ }^{2,3}$ such as visualization agents in magnetic resonance imaging, ${ }^{4}$ therapeutic carriers in drug delivery, 5,6 heat intermediaries in cancer treatment therapies, ${ }^{7,8}$ and labelers for in vitro and in vivo separation experiments. ${ }^{9}$ The usefulness of the magnetic colloids for biomedical application depends on their biocompatibility, the stability of the magnetic nanoparticles in solution at neutral pHs, and the capability of MNP surfaces to become chemically functionalized.

Among many studied materials, Fe and its oxides are the unique FDA (US Food and Drug Admistration) accepted ones. The amphoteric surface of $\mathrm{Fe}$ oxides facilitates their funtionalization. Subsequently, many protocols have been developed for its preparation by either high-temperature decomposition of an organic iron precursor or low-temperature coprecipitation. ${ }^{10}$ The MNPs produced by high-temperature decomposition methodology display better structural and magnetic properties but also hydrophobicity, and further work on MNP surfaces for their stabilization in neutral conditions is needed. Stability in suspension is a major requirement for any biomedical application that involves injection in a living human being and results from the interplay between attractive dipolar and van der Waals interactions and repulsive electrostatic and steric interactions.

Steric stabilization includes coating with nonionic surfactant molecules, polymers, and inorganic layers as silica or some noble metals. ${ }^{11}$ Among the routes employed for obtaining the water-soluble functionalized iron oxide MNPs, the addition of small biocompatible organic molecules, such as amino acids, ${ }^{12}$ peptides, ${ }^{13}$ citric acid, ${ }^{14-16}$ and cyclodextrin, ${ }^{17}$ presents the advantages of combining electrostatic and steric stabilization, assuring coating biocompatibility and providing functionality for biomolecule conjugation. Comparing polymeric stabilization with electrostatic one presents the advantage of reversibility from the aggregated state by dilution but usually involves the binding of a large macromolecule resulting in a coated MNP with a large overall size which strongly modifies its hydrodynamic behavior. So, a renewed interest has appeared in iron oxide nanoparticles synthesized by coprecipitation and

Received: November 23, 2012

Revised: February 22, 2013

Published: February 22, 2013 
Table 1. Synthesis, Magnetic and Structural Characterization Parameters, and Specific Absorption Rate (SAR) under a Radiofrequency Field of $265 \mathrm{kHz}$ and $40.1 \mathrm{kA} / \mathrm{m}$ of Magnetite Aqueous Colloids, Stable in Suspension under Moderate Magnetic Fields ${ }^{a}$

\begin{tabular}{|c|c|c|c|c|c|c|c|c|c|c|c|c|}
\hline & {$[\mathrm{X}](\mathrm{g} / \mathrm{L})$} & $\mathrm{pH}_{\mathrm{ads}} \pm 0.01$ & $\mathrm{pH}_{\text {sus }} \pm 0.01$ & $M_{\mathrm{s}}\left(\mathrm{Am}^{2} / \mathrm{kg}\right)$ & $d_{\mathrm{m}}(\mathrm{nm})$ & $\sigma_{d m}$ & $\delta(\mathrm{nm})$ & $d_{\mathrm{TEM}}(\mathrm{nm})$ & $d_{\mathrm{H}}(\mathrm{nm})$ & $T_{\mathrm{B} i}(\mathrm{~K})$ & $T_{\mathrm{B}}(\mathrm{K})$ & SAR $(W / g)$ \\
\hline $\mathrm{CS}_{1}$ & 13.4 & 4.58 & 7.44 & 16.0 & 7.1 & 2.5 & 5.3 & $9.5 \pm 1.6$ & 18.3 & 81 & 28 & 14.4 \\
\hline $\mathrm{CS}_{2}$ & 10.7 & 4.91 & 7.34 & 9.7 & 7.0 & 2.4 & 7.5 & $8.2 \pm 2.4$ & 17.8 & 92 & 41 & 4.29 \\
\hline $\mathrm{CS}_{3}$ & 18.3 & 5.50 & 6.97 & 24.9 & 6.0 & 2.8 & 3.1 & $9.3 \pm 1.7$ & 26.6 & 166 & 13 & 28.0 \\
\hline $\mathrm{CS}_{4}$ & 13.1 & 6.25 & 7.22 & 26.0 & 7.1 & 3.4 & 3.4 & $11.9 \pm 3.5$ & 23.4 & 196 & 18 & 41.5 \\
\hline $\mathrm{CS}_{5}$ & 5.6 & 6.88 & 7.10 & 17.7 & 5.8 & 2.5 & 4.0 & $9.4 \pm 2.0$ & 30.2 & 132 & 16 & 12.1 \\
\hline $\mathrm{CS}_{6}$ & 7.4 & 7.08 & 7.22 & 11.5 & 6.7 & 2.4 & 6.4 & $10.9 \pm 1.8$ & 20.2 & 126 & 32 & 5.2 \\
\hline
\end{tabular}

${ }^{a} \mathrm{CS}_{i}(i=1-6)$ labeled colloids synthesized by one-step coprecipitation at $60{ }^{\circ} \mathrm{C}$, followed by citric acid adsorption at $\mathrm{pH}_{\text {ads }}$ medium acidity and finally suspended at $\mathrm{pH}_{\text {susp. }}[\mathrm{X}]$ stands for concentration as mass of magnetite per solution volume; typical error is \pm 0.2 . Data derived from $S Q U I D-$ DC magnetometry: $M_{\mathrm{s}}$ stands for specific saturation magnetization, $d_{\mathrm{m}}$ for magnetic core diameter, $\sigma_{d m}$ for standard deviation, and $\delta$ for twice the magnetically frustrated layer thickness. $d_{\mathrm{TEM}}$ stands for nanoparticle diameter retrieved from TEM images, $d_{\mathrm{H}}$ for weight (averaged nanoparticle hydrodynamic diameter), $T_{\mathrm{B} i}$ for blocking temperatures determined from $\mathrm{ZFC}$ curve maximum, $T_{\mathrm{B}}$ for blocking temperature for same size noninteracting nanoparticles, and SAR for Specific Absorption Rate at $40.1 \mathrm{kA} / \mathrm{m}$ and $265 \mathrm{kHz}$ in Watts per gram of magnetite.

stabilized by functionalization of their surface using small molecules with large deprotonation ability like organic polyprotic acids, leading to electrosteric stabilization.

Citric acid $\left(\mathrm{AH}_{3}\right.$ with $\left.\mathrm{A}=\mathrm{C}_{6} \mathrm{H}_{5} \mathrm{O}_{7}\right)$ is a small molecule that has three carboxyl and one hydroxyl groups and is known to chemisorb to the iron oxide nanoparticle by forming a carboxylate group with the $\mathrm{Fe}-\mathrm{OH}$ molecules present on the nanoparticle surface, leaving one or two carboxyl groups negatively charged that can be used for other purposes. A positively charged drug ${ }^{16}$ and/or a fluorescent molecule, like for instance rhodamine, ${ }^{18}$ can be bonded to them for applications in drug delivery or for in vitro labeling studies, respectively. As the $\mathrm{AH}_{3}$ molecule is small the nanoparticles' hydrodynamic radii are not much enlarged, which is of importance in magnetic hyperthermia therapy applications. Differences evidenced in the rheological and microstructural properties of biocompatible citric acid coated magnetites prepared by different protocols based on the coprecipitation method have been recently discussed elsewhere. ${ }^{15}$

Magnetic Fluid Hyperthermia is a therapy to treat cancer tumors. Briefly, MNPs exposed to a radio frequency (rf) field absorb energy from the applied field and dissipate this energy through magnetic relaxation effects locally raising the tumor temperature above $43{ }^{\circ} \mathrm{C}$ and selectively killing tumor cells. ${ }^{7}$ The figure of merit of a given nanoparticle for this application is given by the specific absorption rate (SAR), which is defined as the heat power absorbed from the rf field per gram of magnetic material. Nowadays, active research is being executed on MNPs synthesis protocol to improve specific absorption rates to reduce the amount of material that has to be incorporated into a given tumor for its treatment and also to extend this kind of therapy to smaller tumors, which requires the attainment of larger SAR values. For single-domain MNPs, relaxation processes are of the Néel and Brown types. These processes correspond to the nanoparticle magnetic moment switching among its easy axis directions and the viscous friction due to the Brownian rotational diffusion of particles in the fluid, respectively. As both processes take place in parallel, the heating is driven by the one having the shorter characteristic time. ${ }^{19}$ From the stabilization ways previously outlined emerges the possibility of either using MNPs with large hydrodynamic sizes (polymeric like coating) possibly resulting in a Neel driven process or using MNPs covered with small organic molecules, as the ones studied here, which may dissipate through a Brown type process. The question of which of these mechanisms optimizes the heating process is still an open question, whose answer requires more experimental research.

In this work, highly stable and biocompatible magnetic suspensions of citric acid coated $\mathrm{Fe}_{3} \mathrm{O}_{4}$ nanoparticles in water were produced as biomedical colloids suitable for energy dissipation under an external ac magnetic field in the rf range and appropriated for magnetic hyperthermia therapy. Moreover, the specific heating efficiency at a frequency of $265 \mathrm{kHz}$ and field amplitudes of 20, 27.8, and $40.1 \mathrm{kA} / \mathrm{m}$ were determined and are discussed here in terms of the MNP structural and magnetic parameters. Optimum synthesis conditions for long-term suspension stability, as well as the performance of the obtained ferrofluids for $\mathrm{rf}$ heating, are determined for a wide range of experimental conditions. A detailed analysis of SAR measured values in terms of magnetic and structural colloid properties is addressed. Finally, the coated MNPs were efficiently internalized by human lung adenocarcinoma A549 cells, and its viability was analyzed by flow cytometry using Annexin V - Propidium Iodide (PI) markers.

\section{EXPERIMENTAL DETAILS}

$\mathrm{Fe}_{3} \mathrm{O}_{4}$ nanoparticles (core) were prepared by coprecipitation of ferric chloride and ferrous chloride in the presence of excess ammonia $\mathrm{NH}_{4} \mathrm{OH}$ solution (AS) via a modified Massart method. $^{20,21}$ The so obtained magnetite cores were negatively charged by CA adsorption over its surfaces. The coating was performed without leaving the wet route, to avoid nanoparticle surface passivation. Both steps, coprecipitation and CA adsorption, were carried out under a $\mathrm{N}_{2}$ reflux, to ensure anaerobic conditions, with continuous and vigorous magnetic stirring to ensure reagent homogeneity, and at controlled constant temperature equal to $60{ }^{\circ} \mathrm{C}$. The heating device was close loop controlled to ensure temperature homogeneity and reproducibility.

Briefly, $2.75 \mathrm{~g}$ of $\mathrm{FeCl}_{3} \cdot 4 \mathrm{H}_{2} \mathrm{O}$ and $1.01 \mathrm{~g}$ of $\mathrm{FeCl}_{2} \cdot 6 \mathrm{H}_{2} \mathrm{O}$ were dissolved in $50 \mathrm{~mL}$ of bidistilled water each, mixed in a threeneck flask, and heated to the reaction temperature. Then, $3 \mathrm{~mL}$ of AS $(25 \% \mathrm{w} / \mathrm{w})$ was added drop by drop and left to react for $30 \mathrm{~min}$; after that, $75 \mathrm{~mL}$ of AS was added at a rate of $1 \mathrm{drop} / \mathrm{s}$ until the solution reached a $\mathrm{pH}$ of 10.5 , high enough to prevent agglomeration due to surface charge. The isoelectric point (IEP) of magnetite is known ${ }^{22}$ to be in the $\mathrm{pH}$ range from 6 to 7 depending on the $\mathrm{Fe}^{2+}$ concentration and temperature. Then, the black precipitate was separated from the dispersion medium 
by means of a permanent magnet, mixed with a CA aqueous solution $(0.02 \mathrm{~g} / \mathrm{mL})$, and left to react at $60{ }^{\circ} \mathrm{C}$ during $90 \mathrm{~min}$ to obtain citric acid coated magnetite. To yield uncoated magnetite $(u)$, the black precipitate was washed several times and resuspended in water at a $\mathrm{pH}$ close to neutral (7-7.4).

The $\mathrm{pH}$ at which $\mathrm{CA}$ was adsorbed to the MNP surface $\left(\mathrm{pH}_{\mathrm{ad}}\right)$ was varied from 4.58 to 7.08 . AS $(0.25 \% \mathrm{w} / \mathrm{w})$ was used to adjust the suspension $\mathrm{pH}$ to $\mathrm{pH}_{\text {sus }}$ close to 7 . Then the suspension was again placed in a permanent magnet during 600 s. By this way six colloids $C_{i}$ (with $i=1$ to 6 for increasing $\mathrm{pH}_{\mathrm{ad}}$ ) were prepared. Each one of them was then divided into two parts: the suspension of MNPs that were immobilized with a permanent magnet $\left(\mathrm{CP}_{i}\right)$ and the suspension of the MNPs which remained suspended under the moderate magnetic field created by the magnet $\left(\mathrm{CS}_{i}\right)$. Details about the preparation of the six $C S_{i}$ colloids of $\mathrm{Fe}_{3} \mathrm{O}_{4}$ nanoparticles coated at different $\mathrm{pH}_{\mathrm{ad}}$ are given in Table 1. $\mathrm{pH}$ measurements were carried out with a $\mathrm{pH}$-meter with $\pm 0.01 \mathrm{pH}$ accuracy.

Common and standardized chemical volumetric analysis was used for colloid concentration $[\mathrm{x}]$ determination, expressed as magnetite mass per solvent volume with an accuracy of $2 \%$. $\mathrm{K}_{2} \mathrm{Cr}_{2} \mathrm{O}_{7}$ was used as titrant.

Phase identification and core particle characterization were performed by X-ray diffraction (XRD) and transmission electron microscopy (TEM) on dried samples. Dried coating easily hydrates, hindering sample preparation for XRD analysis, especially in the more efficiently coated cases. The patterns were determined with an X'Pert Diffractometer within a $2 \theta$ range from 20 to $80^{\circ}$. TEM images were obtained with a TEM JEOL JEM 2100 microscope.

Zeta-potential measurements and thermogravimetry (TG) combined with differential thermal analysis (DTA) were used to confirm coating achievement. Measurements were carried out on dried powder samples with a Shimatzu TG-50 and DTA-50 system. During measurements the samples were kept inside platinum crucibles and heated at a constant rate of $10 \mathrm{~K} /$ min under a flux of $50 \mathrm{~mL} / \mathrm{min}$ of $\mathrm{N}_{2}$. The laser doppler electrophoretic method was used to measure zeta potentials $(\zeta$ $[\mathrm{mV}])$ of $1 \mathrm{~mL}$ of colloid with a Malvern Zetasizer nano ZS90 device. The zeta potential was obtained by the application of the Henry equation using the Hückel approximation.

Dynamic light scattering (DLS) experiments were performed on $\mathrm{CS}_{i}$ colloids at a scattering angle of $90^{\circ} \mathrm{C}$ with a goniometer ALV/CGS-5022F with Multiple Tau digital correlator ALV$5000 / E P P$. The light source was a helium-neon laser operating at $22 \mathrm{nW}$.

Specific magnetization $(M)$ as a function of applied magnetic field $(H)$ at room temperature was obtained using a VSM LakeShore 7404 vibrating sample magnetometer operated with a maximum applied field $\mu_{0} H_{\max }=1.5 \mathrm{~T}$ or with a SQUID Quantum Design magnetometer with $\mu_{0} H_{\max }=2.5 \mathrm{~T}$. The latter was also used to measure the magnetization temperature dependence under zero field cooled (ZFC) and field cooled (FC) protocols carried out at $2 \mathrm{~K} / \mathrm{min}$ and $H_{\mathrm{FC}}=100 \mathrm{Oe}$.

Hydrodynamic sizes were obtained from the inverse Laplace transformation of the measured time correlation function assuming the Stokes-Einstein relation between relaxation times and hydrodynamic diameters. ${ }^{23}$ Each sample was measured 10 times. Mean values $\left(d_{\mathrm{H}}\right)$ of the weight-average hydrodynamic sizes are reported in Table 1 .

Time-dependent calorimetric experiments were conducted exposing $1 \mathrm{~mL}$ of the colloid, held in a clear glass Dewar, to $\mathrm{rf}$ fields of $20,27.8$, and $40.1 \mathrm{kA} / \mathrm{m}$ amplitude and $265 \mathrm{kHz}$ frequency. The temperature was sensed during treatment with a fiber optic sensor placed at the center of the sample. The sensor was connected to a calibrated signal conditioner (Neoptix) with an accuracy of $\pm 0.1 \mathrm{C}$. Colloid temperature was kept below 65 ${ }^{\circ} \mathrm{C}$ to minimize evaporation and prevent colloid destabilization.

The SAR parameter was obtained from the initial slope of the heating curve with the expression

$$
\mathrm{SAR}=\frac{C}{[\mathrm{X}]} \frac{\partial T}{\partial t}
$$

where $C$ is the volumetric heat capacity of the solvent $(C=4.18$ $\mathrm{J} / \mathrm{K} \mathrm{cm}^{3}$ ) and $[\mathrm{X}]$ is the MNP concentration given as mass of $\mathrm{Fe}_{3} \mathrm{O}_{4}$ per solvent volume unit.

Viability and discrimination of apoptotic and necrotic cells were determined by double-labeling for the Annexin V and PI assay and analyzed by flow cytometry using a PARTEC PAS III cytometer. It is known ${ }^{24}$ that the plasmatic membrane loses phospholipid asymmetry in the apoptosis, resulting in the exposure of phosphatidylserine (PS) residues at the outer plasma membrane leaflet. Annexin V interacts strongly and specifically with PS, and it is used to detect apoptosis by targeting for this asymmetry. Annexin $\mathrm{V}$ does not bind to normal live cells since the molecule is not able to penetrate the phospholipid bilayer. In necrotic cells the integrity of the plasma membrane is lost, and then Annexin V is able to bind to the cell. To discriminate between necrotic and apoptotic cells PI (a membrane-impermeable DNA stain) is simultaneouly used.

A549 cells were grown as monolayer in Dulbecco's modified Eagle's medium (DMEM) supplemented with 10 vol \% fetal bovine serum and $0.5 \mathrm{mg} \mathrm{mL}^{-1}$ of streptomycin in a humidified $5 \% \mathrm{CO}_{2}$ atmosphere at $37{ }^{\circ} \mathrm{C}$. For the MNP uptake, the cultures at $60 \%$ confluence ( $24 \mathrm{~h}$ incubation time) were washed with phosphate buffered saline (PBS) and were incubated, in 18 $\mathrm{mL}$ of DMEM doped with three distinct concentrations of 34, 67 , and $135 \mu \mathrm{g}_{\mathrm{Fe} 3 \mathrm{O} 4} / \mathrm{mL}$ of culture medium, in a $75 \mathrm{~cm}^{2}$ flask during $12 \mathrm{~h}$. Then, cells were washed four times with PBS to remove the nonincorporated MNPs and trypsinized from each flask. From each A549 internalized culture, $10^{6}$ cells were resuspended in Annexin-binding buffer and stained with $5 \mu \mathrm{L}$ of Annexin and $5 \mu \mathrm{L}$ of propidium iodide. Cells incubated without nanoparticles at the same condition of the internalized ones ( $36 \mathrm{~h}$ incubation time) were used as control.

\section{RESULTS AND DISCUSSIONS}

3.1. Stability Analysis. The crucial step in the preparation of a stable aqueous suspension of MNPs resides on their surfaces engineering, in this case on the CA adsorption efficiency. Representative DTA-TG curves of citric acid and the $\mathrm{CS}_{i}$ colloid are shown in Figure 1a and b, respectively. The DTA curve in Figure 1(a) displays a sharp endothermic peak at $157{ }^{\circ} \mathrm{C}$ without weight loss at the corresponding TG, assigned to fusion $\left(\Delta H_{\text {fus }}=-0.14 \mathrm{~kJ} / \mathrm{g}\right)$, and a broad endothermic feature (centered at $217{ }^{\circ} \mathrm{C}$ ), assigned to citric acid decomposition $(\Delta H=-0.55 \mathrm{~kJ} / \mathrm{g})$, happening in many steps. TG data show an abrupt single-step weight loss (onset at $209{ }^{\circ} \mathrm{C}$ ) of $87.5 \%$ due to CA decomposition. On the other hand, no fusion peak appears at the DTA curve (Figure 1b) of coated nanoparticles, indicating the absence of free CA precipitation, and exothermic peaks related to magnetite oxidation commencing around $200{ }^{\circ} \mathrm{C}$ are superimposed to attached CA decomposition. The TG curve displays a two-step 


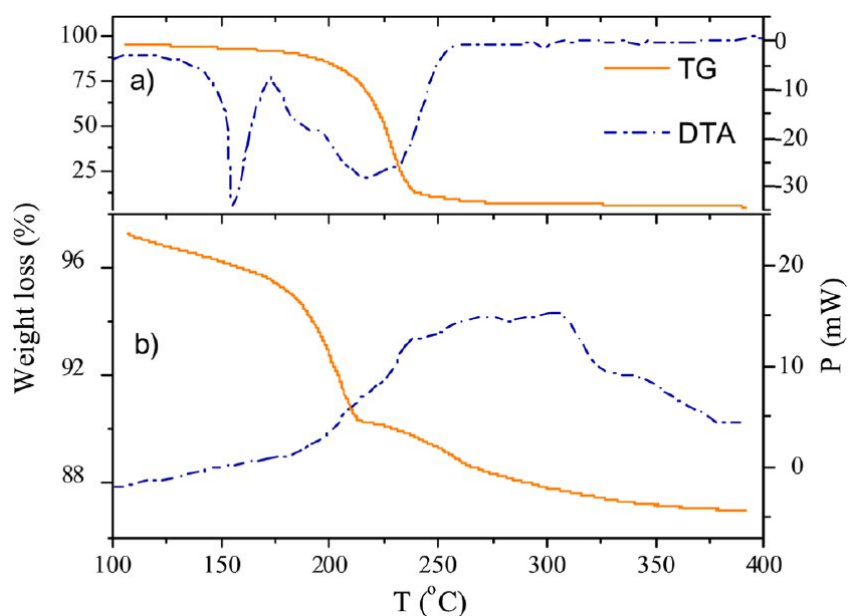

Figure 1. TG and DTA plots of neat citric acid at the upper part (a) and $\mathrm{CS}_{i}$ colloid at the lower part (b).

weight loss (onsets at 175 and $222^{\circ} \mathrm{C}$ ) of 7.3 and $2.5 \%$ which is attributed to desorption of citric acid molecules from the nanoparticle surface. ${ }^{18}$ Two-step weight loss is an indication of a bilayer coating formation: at the first layer the CA molecules are chemically attached to the MNP surface and the second is bound to the first through hydrogen bonds. The latter desorbs at lower temperature. Two-step weight loss has been observed at the MNP coated by bilayers of $n$-alkanoic acids. ${ }^{25}$ From the comparison of these thermogravimetric curves it is inferred that the binding of the CA molecule to the MNP surface was accomplished.

Both types of aqueous suspensions $\left(\mathrm{CP}_{i}\right.$ and $\left.\mathrm{CS}_{i}\right)$ are very stable, and the MNPs remain suspended for periods of several months, $\mathrm{CS}_{i}$ colloids being extremely stable. For $\mathrm{CS}_{i}$ colloids the separation of the MNPs from the dispersion media was not even possible by three times centrifugation at $13000 \mathrm{rpm}$ during $600 \mathrm{~s}$. The mass fraction $(f)$ of suspended nanoparticles, defined as the quotient of concentrations, measured at $\mathrm{pH}_{\text {sus }} f$ $=\left[\mathrm{CS}_{i}\right] /\left[\mathrm{C}_{i}\right]$, was used as a parameter to quantify the CA adsorption efficiency and as a long-term stability criterion. In Figure 2, it can be seen that $f$ decreases monotonically with $\mathrm{pH}_{\mathrm{ads}}$. The aqueous citric acid solution left to react with the oxide particles during the synthesis consists of a $\mathrm{pH}$-dependent mixture of $\mathrm{AH}_{3}, \mathrm{AH}_{2}^{-}, \mathrm{AH}^{2-}$, and $\mathrm{A}^{3-}$. The molar fractions $x_{\mathrm{y}}$ ( $\mathrm{y}=\mathrm{AH}_{3}, \mathrm{AH}_{2}^{-}, \mathrm{AH}^{2-}$, and $\mathrm{A}^{3-}$ ) calculated using the known acid dissociation constants $\mathrm{p} K_{\mathrm{a} 1}=3.13, \mathrm{p} K_{\mathrm{a} 2}=4.76$, and $\mathrm{p} K_{\mathrm{a} 3}=$ 6.40 are included in Figure 2. The larger $f$ value is achieved for CA adsorption at $\mathrm{pH}_{\mathrm{ads}}=4.58$, when $60 \%$ of CA molecules were negatively charged as $\mathrm{AH}_{2}^{-}$and $40 \%$ as $\mathrm{AH}^{2-}$. The decrease of $\mathrm{AH}_{2}^{-}$and $\mathrm{AH}^{2-}$ occurring at $\mathrm{pH}$ larger than 5.5 disfavors stability. At $\mathrm{pH}$ values lower than 4.58 , there is a large dissolution rate, and the MNP dissolves during preparation. ${ }^{26}$

Zeta-potential $(\zeta)$ measurements of $\mathrm{CS}_{2}, \mathrm{CS}_{4}, \mathrm{CS}_{6}$, and $u$ colloids are shown in Figure 3, and the mean $\zeta$ values against $\mathrm{pH}$ are displayed in Figure 4. The isoelectric point (IEP) of coated nanoparticles is close to 2 , and for $\mathrm{pH}>4$ the largest mean value of $\zeta$ is close to $-36 \mathrm{mV}$. Results suggest that at larger $\mathrm{pH}_{\text {sus }}$ stability occurs when a larger amount of $\mathrm{AH}_{2}{ }^{-}$is present at binding conditions; i.e., a larger amount of CA binds to the nanoparticle surfaces by one carboxylate leaving two free dangling ends. Further deprotonation of an already bonded molecule occurs at $\mathrm{pH}$ value larger than $\mathrm{p} K_{\mathrm{a} 3}=6.40$, providing electrostatic and steric stabilization. Mean $\zeta$ values as large as

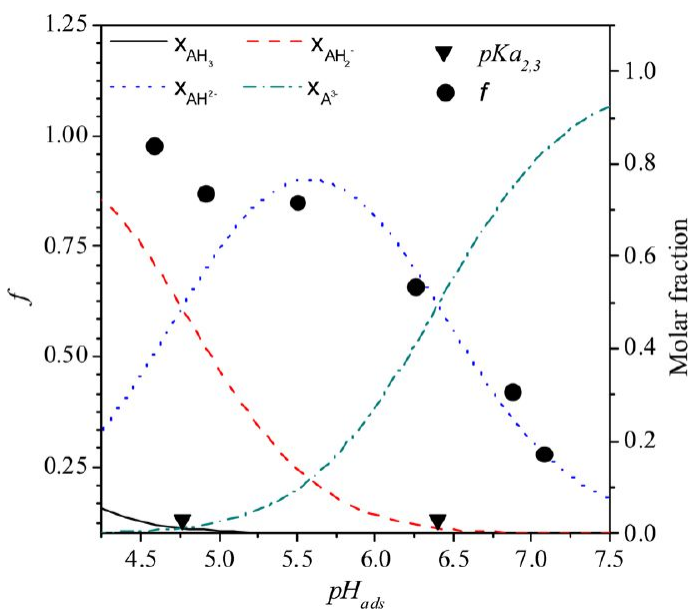

Figure 2. Dots stand for the fraction $(f)$ of MNP that remains stable at neutral $\mathrm{pH}$ after exposure to $0.1 \mathrm{~T}$ during $600 \mathrm{~s}$, used as a long-term stability parameter, triangles for acid dissociation constants $\left(\mathrm{p} K_{\mathrm{a}}\right)$, and $\mathrm{pH}_{\mathrm{ads}}$ for adsorption $\mathrm{pH}$. Lines stand for theoretical calculations of molar fraction of the species composing the aqueous citric acid solution vs adsorption $\mathrm{pH}_{\mathrm{ads}}$ using equilibrium dissociation constants for deprotonation reactions.

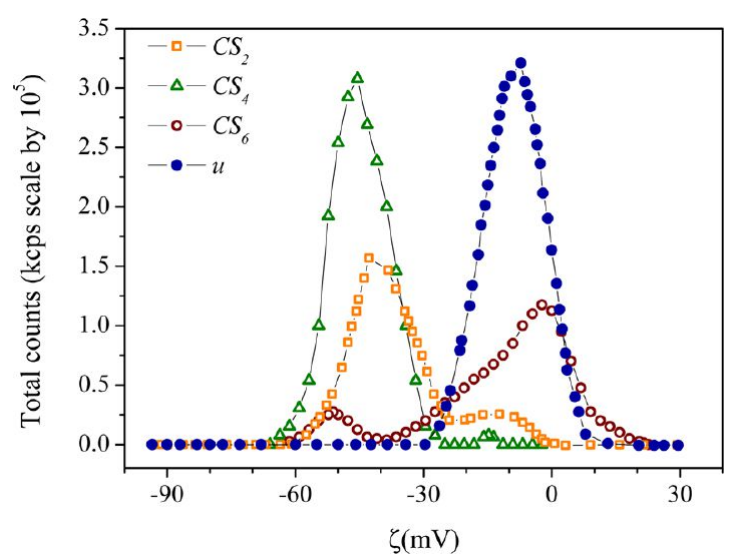

Figure 3. Zeta potential $(\zeta)$ distribution of coated MNPs measured at neutral suspension $\mathrm{pH}$.

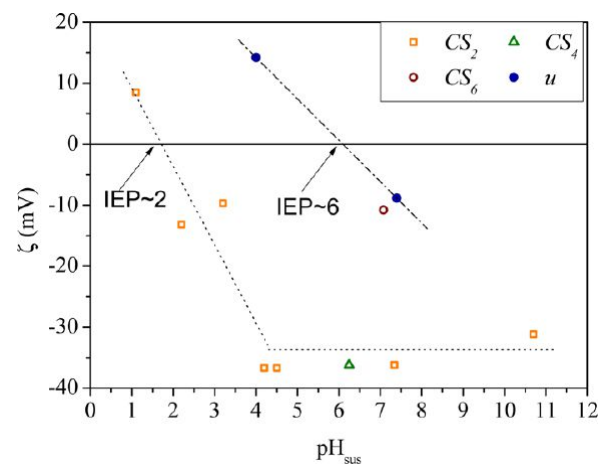

Figure 4. Mean zeta potential $(\zeta)$ against suspension $\mathrm{pH}_{\text {sus. }}$. Lines stand to guide the eye and IEP for the isoelectric point.

$-36 \mathrm{mV}$ confirm that the particles have become highly negatively charged and that the coating was accomplished. Our results differ from a previous work ${ }^{14}$ where the largest amount of magnetic nanoparticles in stable suspension at $\mathrm{pH}_{\text {susp }}$ $=10.1$ was obtained with $\mathrm{CA}$ adsorption at $80^{\circ} \mathrm{C}$ and $\mathrm{pH}_{\mathrm{ads}}=$ 5.2 where $70 \%$ the molecules are twice deprotonated as $\mathrm{AH}^{2-}$ 


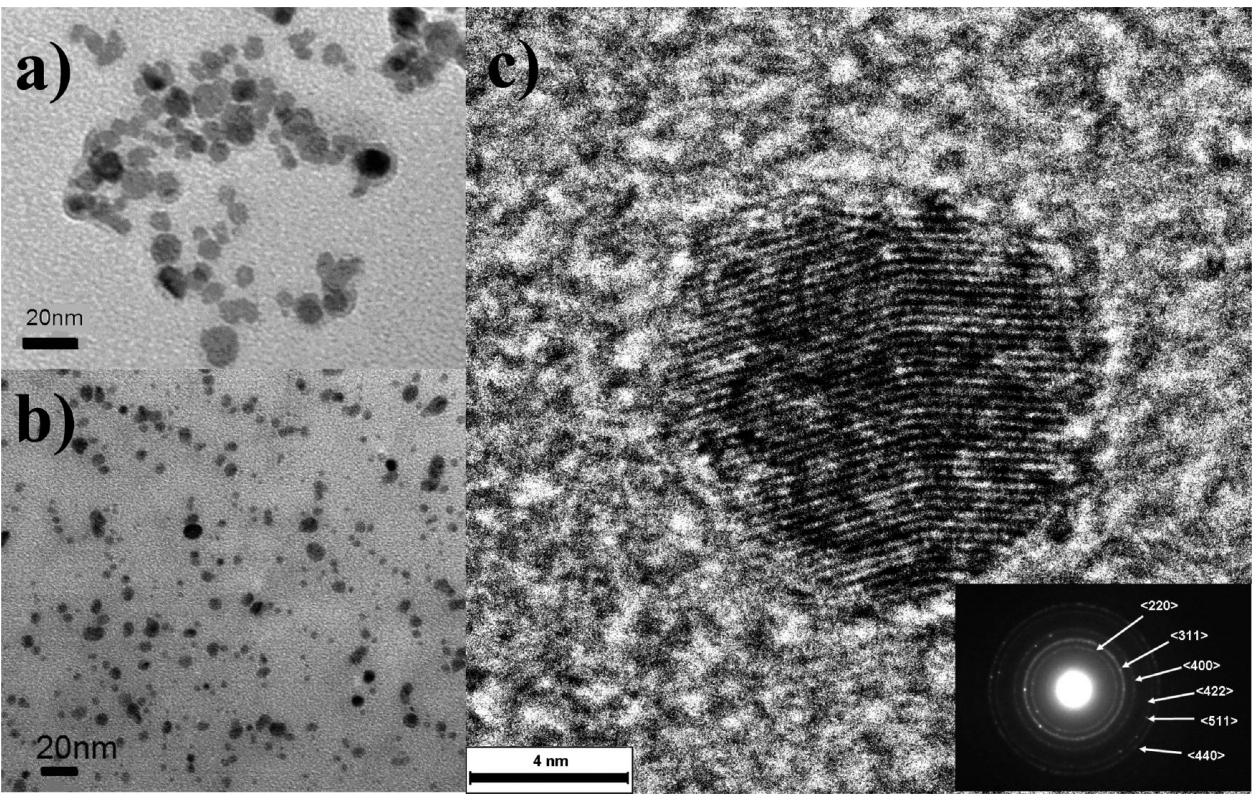

Figure 5. TEM images of uncoated (a) and citric acid coated particles $\left(\mathrm{CS}_{6}\right)(\mathrm{b})$ and isolated particle of $\mathrm{CS}_{6}$ dried colloid (c).

and $25 \%$ once deprotonated; however, the largest $|\zeta|$ determined in both works coincide because both were measured at $\mathrm{pH}$ values larger than $\mathrm{pK}_{\mathrm{a} 3}$ where the three carboxyl groups are dissociated. A largest $\zeta$ value of -25.6 at $\mathrm{pH} 6$ was obtained in other recent work $^{16}$ where coated magnetite was optimized for drug delivery. A lower $\zeta$ value means less charge and may be related to a shorter adsorption time (30 min less).

3.2. Structural and Magnetic Analysis. X-ray diffractograms for $u$ and $\mathrm{CS}_{i}$ samples present typical X-ray diffraction patterns of magnetite (see Figure $\mathrm{S} 1$ in Supporting Information). The diffraction patterns display well-defined peaks belonging to the cubic spinel structure, indicating that the samples are single phase. The whole patterns were refined, including peak broadening due to crystallite size, using a cubic spinel structure (space group $\mathrm{Fd} 3 \mathrm{~m}$ ) with lattice parameter $a=$ $8.378 \AA$ and the $\mathrm{O}$ atoms arranged in a face-centered-cubic lattice. Figure 5a displays a typical TEM image of $u$-MNPs $\left(d_{\mathrm{TEM}}=9.9 \pm 2.4 \mathrm{~nm}\right)$. These particles are not regularly faceted; instead they show spheroid-like forms and are quite aggregated. A representative TEM image of a coated colloid $\left(\mathrm{CS}_{6}\right)$ is shown Figure $5 \mathrm{~b}$ and a single $\mathrm{CS}_{6}$ particle in Figure $5 \mathrm{c}$. The selected area electron diffraction pattern indicates good crystallinity (see inset of Figure 5c). The MNPs are spherical, more uniform, and further apart from each other than uncoated nanoparticles. Chain-like arrangements due to dipolar interactions appear. Mean size values estimated from TEM data are listed in Table 1. $\mathrm{CS}_{i}$ and $\mathrm{CP}_{i}$ colloids display similar mean size; for example, $d_{\text {TEM }}$ for $\mathrm{CP}_{6}$ is $10.8 \pm 2.7 \mathrm{~nm}$.

Magnetic analysis was performed on dried colloid samples and on frozen uncoated and both $\mathrm{CS}_{i}$ and $\mathrm{CP}_{i}$ coated colloids. Each sample is a collection of single domain particles of magnetic volume $V$ and giant magnetic moment $\mu=M_{\mathrm{s}} \rho V$ ( $\rho$ is the mass density). Particle magnetocrystalline anisotropy axes are randomly distributed. The energy of this ensemble placed in a magnetic field $H$ arises from three main contributions, the magnetic anisotropy energy, the particle magnetic moment Zeeman interaction with the applied field, and the dipolar interaction among particles.
The first and third energy terms dominate at low field, and then determine the magnetization behavior as a function of temperature in FC and ZFC curves displayed in Figure 6 for $u$

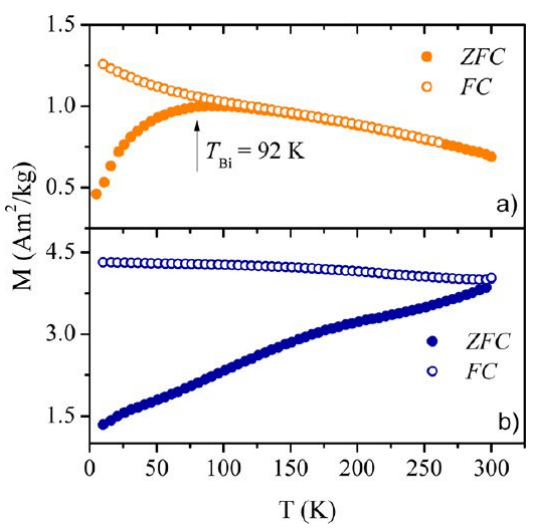

Figure 6. Specific magnetization temperature dependence for zerofield-cooled (ZFC) and field-cooled (FC) protocols for (a) $\mathrm{CS}_{2}$ and (b) uncoated colloids (dried samples), acquired with $H_{\mathrm{FC}}=100 \mathrm{Oe}$ and heating rates of $2 \mathrm{~K} / \mathrm{min}$.

and $\mathrm{CS}_{2}$ samples. The absence of a maximum in the ZFC curve of the $u$ sample indicates a blocking temperature close to room temperature due to aggregation and strong dipolar interaction between particles. The constancy of $M$ almost in the whole FC curve is also a signature of a highly interacting system. The ZFC curve of $\mathrm{CS}_{2}$ exhibits a maximum at the blocking temperature $T_{\mathrm{B} i}=92 \mathrm{~K}$, and $\mathrm{ZFC}$ bifurcates from the $\mathrm{FC}$ curve at the irreversibility temperature $T_{i}=140 \mathrm{~K}$. For temperatures higher than $T_{i}$, magnetization temperature dependence strongly departs from the Curie-like behavior $(M \sim H / T)$ expected for a noninteracting system. As temperature decreases from $T_{i}$ the FC magnetization increases. Comparing ZFC-FC curves of uncoated and coated MNPs it is clear that citric acid coating prevents strong aggregation and lowers dipolar interaction, but still the system behaves as an interacting superparamagnet 
(ISP). Colloids blocking temperatures $T_{\mathrm{B} i}$ obtained from ZFC data acquired from frozen colloids are listed in Table 1 .

The second energy term dominates at high fields and is the main contribution to the hysteresis loops shown in Figure 7.

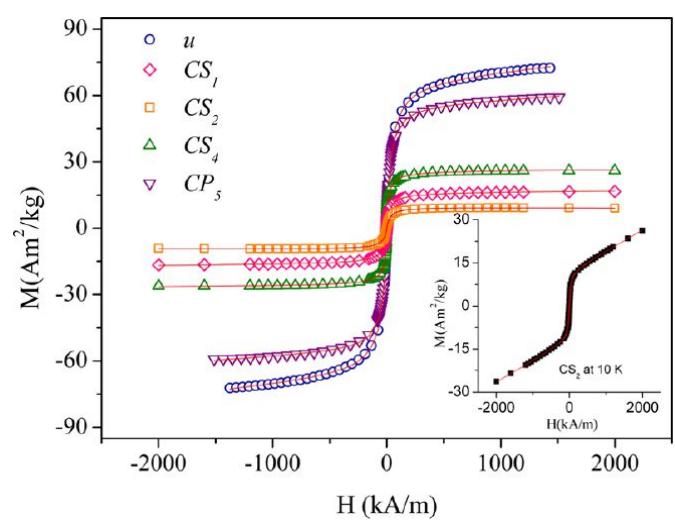

Figure 7. Specific magnetization loops measured at $290 \mathrm{~K}$ for various colloids. Inset: $\mathrm{CS}_{2}$ measurement at $10 \mathrm{~K}$. Solid lines stand for best Langevin fits using eq 2 .

The mean particle magnetic moment $\langle\mu\rangle$ in all the studied samples is around $1.5 \times 10^{4} \mu_{\mathrm{B}}$; the anisotropy energy barrier $K_{\text {eff }} V$ is much smaller than the magnetic moment field interaction energy $\mu_{0} \mu \mathrm{H}$; and the magnetization of the particles at a given temperature and field can be modeled as

$$
M(T, H)=N \int_{0}^{\infty} \mu L\left(\frac{\mu_{0} \mu H}{k_{\mathrm{B}} T}\right) f(\mu) \mathrm{d} \mu+\chi_{a} H
$$

where the Langevin function $L(x)=\operatorname{coth}(x)-1 / x$, being $x=$ $\mu_{0} \mu \mathrm{H} / k T$, is convoluted with a log-normal distribution of magnetic moments $f(\mu)=\exp \left[-\left(\ln \left(\mu / \mu_{\mathrm{m}}\right) / 2 \sigma^{2}\right)\right] / \mu \sigma(2 \pi)^{1 / 2}$, and $\chi_{a}$ is the high field susceptibility ${ }^{27}$ related to surface magnetic disorder induced by the lack of symmetry. Not fully coordinated atoms at the surface of the particle lead to a magnetically frustrated layer which does not saturate even at 10 $\mathrm{K}$ and $2000 \mathrm{kA} / \mathrm{m}$ (see inset in Figure 7). The specific saturation magnetization is $M_{s}=N\langle\mu\rangle=N \int_{0}^{\infty} \mu f(\mu) \mathrm{d} \mu$, where $N$ is the particle number mass density and $\langle\mu\rangle$ the mean magnetic moment. $\chi_{a}$ values are of the order of $10^{-7} \mathrm{~m}^{3} / \mathrm{kg}$. Although the cycles are well fitted with eq 2 and the derived $M_{\mathrm{s}}$ values are correct, the so obtained mean magnetic moments display an unexpected temperature dependence. ${ }^{28}$ In Figure 8 the mean values $\langle\mu\rangle=\mu_{\mathrm{m}} \exp \left(\sigma^{2} / 2\right)$ for various $\mathrm{CS}_{i}$ samples, obtained from best fits of $M$ vs $H$ data of dried colloid powders and frozen colloids, display an increasing behavior with temperature which is characteristic of ISP systems. Then, to determine the particle size distribution from the magnetic properties of each colloid and to compare it with the results obtained by TEM, $\langle\mu\rangle$ is derived from $M$ vs $H$ data acquired from colloidal samples at room temperature (see Figure 7) where the effect of the dipolar interaction on the recorded pattern is diminished. Using $\mu=M_{\mathrm{s}}^{\text {bulk }} \rho V$ with magnetite density $\rho=5175 \mathrm{~kg} / \mathrm{m}^{3}$ and $M_{\mathrm{s}}^{\text {Bulk }}=86 \mathrm{Am}^{2} / \mathrm{kg}$, a lognorm number distribution of magnetic core diameters $f\left(d_{\mathrm{m}}\right)$ (with median $d_{0}=\left(6 \mu_{0} / \pi M_{\mathrm{s}}^{\text {bulk }} \rho\right)^{1 / 3}$ and $\sigma_{d_{\mathrm{m}}}=\sigma / 3$ for spherical particles) is derived from $f(\mu)$ (see Figure 9). From mean $\left\langle d_{\mathrm{m}}\right\rangle$, mean nanoparticle size is derived as $d=\left\langle d_{\mathrm{m}}\right\rangle+\delta$ with $\delta$ $=\left\langle d_{\mathrm{m}}\right\rangle\left(\left(M_{\mathrm{s}}^{\text {bulk }} / M_{\mathrm{s}}\right)^{1 / 3}-1\right)$, twice the magnetically frustrated surface layer thickness. From this analysis, it is concluded that

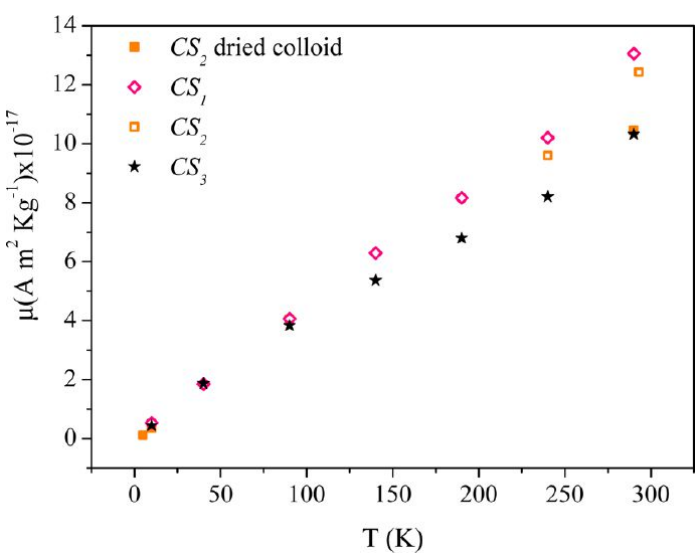

Figure 8. Mean magnetic moment derived from best fits of specific magnetization vs applied field using eq 2, for various frozen colloids, colloids $(290 \mathrm{~K})$, and $\mathrm{CS}_{2}$ dried powder.

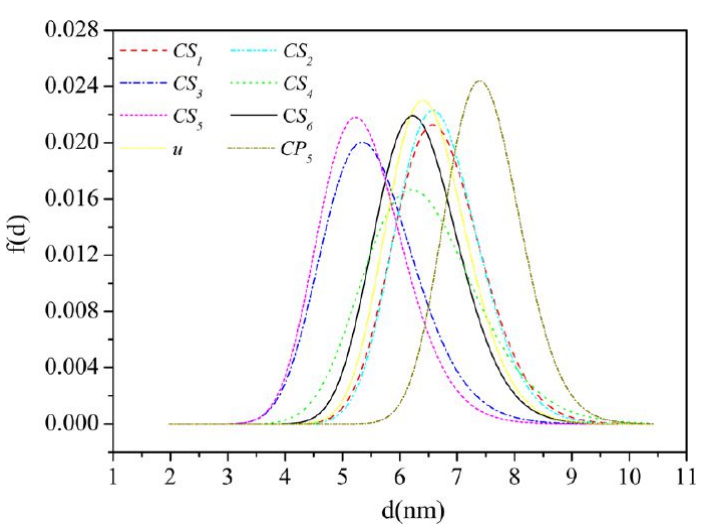

Figure 9. Log norm distribution of magnetic core diameters retrieved from the log norm magnetic moment distribution that fits the data in Figure 7.

the colloids $\mathrm{CS}_{i}$ and $\mathrm{CP}_{i}$ under study here are single domain and behave as interacting superparamagnets. For $\mathrm{CS}_{1}, \mathrm{CS}_{2}$, and $\mathrm{CS}_{6}$ samples the size derived from magnetic cycles is larger than the mean sizes derived by TEM, while for $\mathrm{CS}_{3}, \mathrm{CS}_{4}$, and $\mathrm{CS}_{5}$ the agreement is good. This discrepancy can be ascribed to the fact that TEM, although being a more direct determination, involves a smaller number of particles than the macroscopic magnetic measurement.

A charged particle moving in a solvent drags with it a layer of fluid molecules and counterions, resulting in an entity with a quite large hydrodynamic diameter $d_{\mathrm{H}}$. In effect, in this study it was found that $d_{\mathrm{H}}$ values are 1 order of magnitude larger than the overall nanoparticle size including CA coating (see Table $1)$.

3.3. Specific Absorption Rates (SARs). The heating ability of the various colloids analyzed here was calorimetrically determined, and typical heating curves are shown in Figure 10. The time required to reach a given temperature, at fixed frequency, decreases with increasing field amplitude. SAR represents the power released per gram of magnetite upon acfield application and is mainly given by the magnetization loop area. SAR values listed in Tables 1 and 2 were obtained from the initial slope of the heating curves using eq 1 . These values range from 5.2 to $41.5 \mathrm{~W} / \mathrm{g}$ for $\mathrm{CS}_{i}$ colloids, from 29 to $104 \mathrm{~W} /$ $\mathrm{g}$ for $\mathrm{CP}_{i}$, and $203 \mathrm{~W} / \mathrm{g}$ for the $u$-MNP colloid. The inspection of Figure 9 shows that for our samples there is no clear relation 


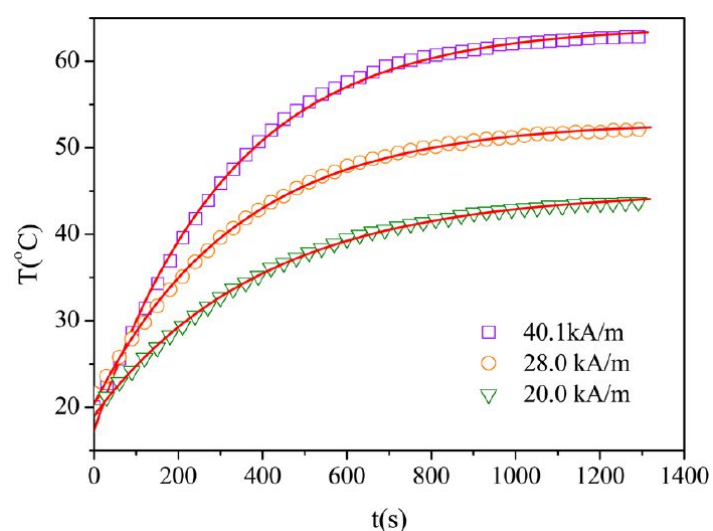

Figure 10. Heating curves acquired at $265 \mathrm{kHz}$ and at various field amplitudes $H_{0}$.

Table 2. Magnetic Characterization and Radiofrequency Dissipation of Magnetite Stable Aqueous Colloids and Uncoated Colloid $^{a}$

\begin{tabular}{lrcrr}
\multicolumn{1}{c}{ colloid } & \multicolumn{1}{c}{$u$} & $\mathrm{CP}_{4}$ & $\mathrm{CP}_{5}$ & \multicolumn{1}{c}{$\mathrm{CP}_{6}$} \\
$\mathrm{SAR}(\mathrm{W} / \mathrm{g})$ & 203 & 28.78 & 93.6 & 104.2 \\
$M_{\mathrm{s}}\left(\mathrm{Am}^{2} / \mathrm{kg}\right)$ & 67.1 & 33.6 & 56.1 & 70.6 \\
$d_{\mathrm{m}}(\mathrm{nm})$ & 6.8 & 7.9 & 7.7 & 8.4 \\
$\delta(\mathrm{nm})$ & 0.6 & 3.2 & 1.2 & 0.6 \\
{$[\mathrm{X}]$} & 8.28 & 6.1 & 10.2 & 6.0
\end{tabular}

${ }^{a} \mathrm{CP}_{i}(i=4,5$, and 6) colloids synthesized as detailed in Table 1 and magnetically separated and $u$ labels uncoated colloid. SAR stands for specific absorption rate at $40.1 \mathrm{kA} / \mathrm{m}$ and $265 \mathrm{kHz}$ in Watts per gram of magnetite. Data derived from SQUID-DC magnetometry: $M_{\mathrm{s}}$ stands for specific saturation magnetization, $d_{\mathrm{m}}$ for magnetic core diameter, and $\delta$ for twice the magnetically frustrated layer thickness.

between SAR values either with magnetic core sizes or with distribution standard deviation $\sigma_{d m}$. For instance, $\mathrm{CS}_{6}, \mathrm{CS}_{4}$, and $u$ colloid dissipate $5.2,41.5$, and $203 \mathrm{~W} / \mathrm{g}$, respectively, and all have nearly the same mean size. The main differences between these samples reside on saturation magnetization and blocking temperatures. Lower saturation magnetizations are assigned to larger magnetically frustrated layers and larger blocking temperatures to interaction and aggregation.

Now we evaluate SAR data in terms of the colloids' physical characterization given by $M_{\mathrm{s}},\left\langle d_{\mathrm{m}}>, \delta, T_{\mathrm{B} i}\right.$ and $d_{\mathrm{H}}$ parameters listed in Tables 1 and 2.

Through the linear response theory the SAR parameter is theoretically given by ${ }^{19}$

$$
\operatorname{SAR}=\frac{\pi \mu_{0} H_{0}^{2} f}{\rho} \int_{0}^{\infty} \chi^{\prime \prime}(\tau(d)) f(d) \mathrm{d} d
$$

where $\mu_{\mathrm{o}}$ is the permeability of free space $\left(4 \pi \times 10^{-7} \mathrm{H} / \mathrm{m}\right)$; $f(d)$ is the size distribution of MNPs having diameters $d$; and $\chi^{\prime \prime}$ is the out-of-phase magnetic susceptibility. SAR field amplitude dependence, plotted in Figure 11, displays an increasing behavior as expected from eq 3. The dependence departs from the linear relation expected for SAR plotted against $\mathrm{H}_{0}{ }^{2}$ for $H_{0}$ values larger than a maximum field. Once this field is exceeded, the area of the hysteresis loop does not change, and SAR remains constant.

For noninteracting nanoparticles, $\chi^{\prime \prime}$ is a function of the relaxation time $(\tau)$ as $\chi^{\prime \prime}(\omega)=\chi_{0}\left((\omega \tau) /\left(1+(\omega \tau)^{2}\right)\right)$, with $\omega=$ $2 \pi f$ and the dc-susceptibility $\chi_{0} \cong\left(\mu_{0} \rho^{2} M_{\mathrm{s}}^{2} V\right) /\left(3 k_{\mathrm{B}} T\right)$, for small magnetic field amplitude. Because the colloids behave as

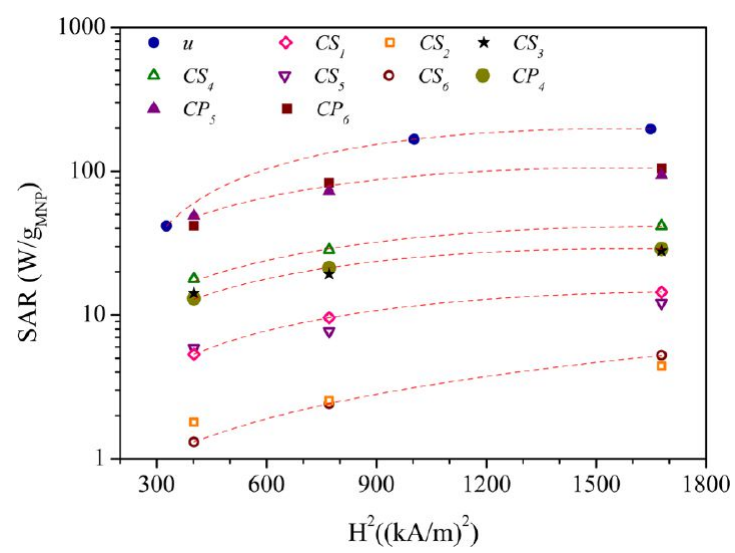

Figure 11. Specific absorption rate (SAR) field amplitude dependence. Dotted lines stand for second-order polynomial fits.

interacting superparamagnets, the dynamics of such systems strongly depends on many physical parameters related with the MNP properties and on the coupling strength. Whether the relaxation is driven by Brown $\left(\tau_{\mathrm{B}}=\left(3 \eta V_{\mathrm{H}}\right) /\left(k_{\mathrm{B}} T\right)\right)$ or by Néel $\left(\tau_{\mathrm{N}}\right)$ mechanisms depends on liquid carrier viscosity $\eta$, hydrodynamic particle volume $\left(V_{\mathrm{H}}\right)$, magnetic energy barrier $E_{\mathrm{a}}$, and attempt time $\tau_{0}$, both $E_{\mathrm{a}}$ and $\tau_{0}$ being size dependent. ${ }^{29}$ The processes take place in parallel $\left(\tau^{-1}=\tau_{\mathrm{B}}{ }^{-1}+\tau_{\mathrm{N}}{ }^{-1}\right)$ and prevails the one having the shorter relaxation time.

$\tau_{\mathrm{B}}$ values calculated at $300 \mathrm{~K}$ using data listed in Table 1 and $\eta=8.90 \times 10^{-4} \mathrm{~Pa}$ s for water fall in the range $0.2 \times 10^{-5}$ to 0.9 $\times 10^{-5} \mathrm{~s}$ and are plotted vs SAR in Figure 12 .

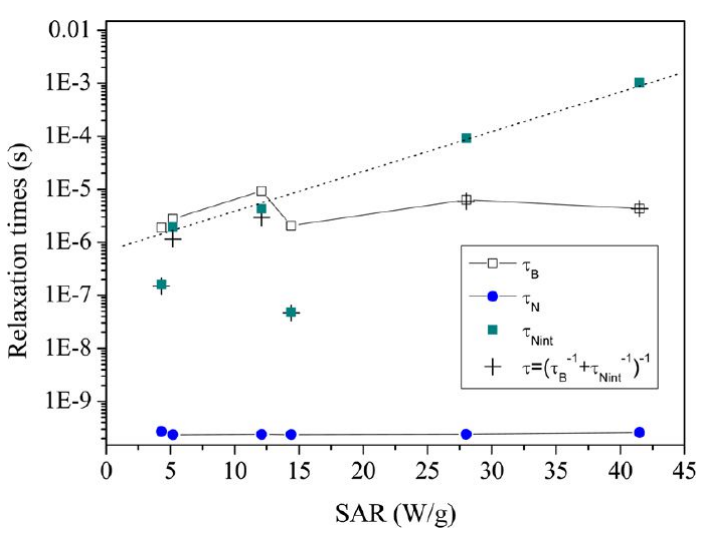

Figure 12. Brown relaxation times $\tau_{\mathrm{B}}=\left(3 \eta V_{\mathrm{H}}\right) /\left(k_{\mathrm{B}} T\right)$ calculated with $k_{\mathrm{B}}=1.38 \times 10^{-23} \mathrm{~J} \mathrm{~K}^{-1}$, viscosity $\eta=8.90 \times 10^{-4} \mathrm{~Pa} \mathrm{~s}$, and $T=300 \mathrm{~K}$. Néel relaxation times $\tau_{\mathrm{N}}=\tau_{0} \exp \left(k_{\text {eff }} V / k T\right)$ of isolated nanoparticles calculated using size-dependent anisotropy energy density $k_{\text {eff }}(d)$ and $\tau_{0}(d)$ interpolated from data in refs 31 and 32 . Relaxation times $\tau_{\text {Nint }}$ for the Néel mechanism taking into account size dependence and interaction.

$\tau_{\mathrm{N}}$ for isolating particles is given by ${ }^{30}$ by

$$
\tau_{\mathrm{N}}=\tau_{0} \exp (\sigma) \quad \text { with } \quad \sigma_{\mathrm{a}}=E_{\mathrm{a}} / k_{\mathrm{B}} T
$$

The energy $E_{\mathrm{a}}$ of isolated particles is equal to the magnetic anisotropy energy $K_{\text {eff }} V$, and $K_{\text {eff }}$ is the size-dependent effective anisotropy energy density. For spherical particles, assuming that the overall anisotropy of the particle is uniaxial, the relation $K_{\text {eff }}(d)=K_{\text {bulk }}+(6 / d) K_{\mathrm{s}}$ is commonly used, where $K_{\text {bulk }}=1 \times$ $10^{4} \mathrm{~J} / \mathrm{m}^{3}$ is the bulk anisotropy energy density of magnetite at room temperature and $K_{\mathrm{s}}$ is the surface anisotropy density. This 
$K_{\text {eff }}(d)$ relation models the increase of the anisotropy energy density with decreasing particle size and has been tested by Gilmore et al. $^{31}$ in magnetite particles grown inside three protein cages of distinct size, where the cage served to constrain particle size and shape and to mute interparticle interaction. Using this relation with $K_{s}(d)=2 \tanh (d / 4)$, as in ref 31 , we have retrieved $K_{\text {eff }}$ for each particle size listed in Table 1 . The corresponding $\tau_{0}$ values were interpolated from the $\tau_{0}(d)$ relation built from data in refs 31 and 32 . Evaluation of $\tau_{\mathrm{N}}$ disregarding interactions results in values in the range $0.1-0.6$ $\times 10^{-9} \mathrm{~s}$.

To take into account that the magnetic moments of interacting particles fluctuate with different relaxation times than those of isolated ones, a total energy barrier given by $E_{\mathrm{a}}=$ $K_{\text {eff }} V+E_{\text {int }}$ was assumed. ${ }^{33}$ The interaction energy $E_{\text {int }}$ was deduced from the shift of the ZFC maximum (Figure 6), disregarding $\tau_{0}$ dependence with interactions, with respect to a noninteracting ensemble as $E_{\text {int }}=k_{\mathrm{B}}\left(T_{\text {Bint }}-T_{\mathrm{B}}\right) \ln \left(t_{\mathrm{m}} / \tau_{0}\right)$. Here $T_{\mathrm{B}}$ is the blocking temperature expected for noninteracting particles of sizes listed in Table 1 , as derived from the $K_{\text {eff }}$ and $\tau_{0}$ values mentioned above; $T_{\text {Bint }}$ is the blocking temperature resulting from ZFC measurements (see Table 1); and $t_{\mathrm{m}} \sim 100$ $s$ is the SQUID measuring time. Then, Néel relaxation time for interacting particles $\tau_{\text {Nint }}$ was obtained with eq 4 with $\sigma=\left(K_{\text {eff }} V\right.$ $\left.+E_{\text {int }}\right)$. The results are shown in Figure 12 where the relaxation times are plotted vs SAR values determined with $H_{0}=40.1 \mathrm{kA} /$ m, i.e., maximum used $H_{\mathrm{o}}$ value, larger than the anisotropy field. Interaction results in slower magnetic relaxation. A crossover between $\tau_{\text {Nint }}$ and $\tau_{\mathrm{B}}$ behavior appears at $12.8 \mathrm{~W} / \mathrm{g}$. The larger SAR values come out with the more interacting colloids, i.e., for the particles displaying larger $T_{\mathrm{B} i}$ (large $E_{\text {int }}$ values). In those cases the process seems to be driven by a Brown mechanism, while for the other cases the Néel mechanism prevails.

The uncoated magnetite results in a very unstable suspension but produces the largest SAR of $200 \mathrm{~W} / \mathrm{g}$. Although particle size is similar to those of coated particles, the hydrodynamic size is $1.5 \mu \mathrm{m}$ (polydispersity index $\sigma_{\mathrm{PI}}=0.5$ ) indicating the presence of large clusters of particles moving in the fluid. Blocking temperature is around $300 \mathrm{~K}$, due to strong interactions. The analysis outlined above for coated colloids, when applied to the uncoated colloid, results in relaxation times various orders of magnitude larger. Still $\tau_{\mathrm{B}}<\tau_{\text {Nint }}$, supporting the idea that interactions play an important role in magnetic fluid hyperthermia increasing nanoparticle energy dissipation.

SAR values against $M_{s}$ are plotted in Figure 13 for all $\mathrm{CP}_{i}$, $\mathrm{CS}_{i}$, and $u$ colloids. An increasing behavior is observed, as

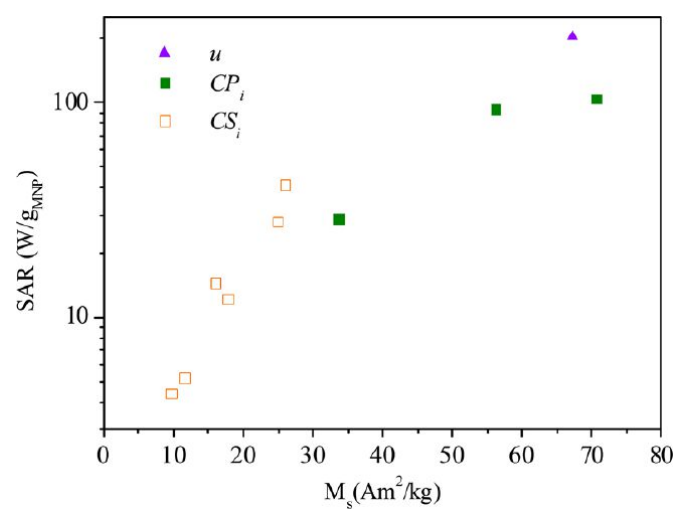

Figure 13. SAR dependence with specific saturation magnetization. expected from the $\chi_{0}$ dependence with $M_{\mathrm{s}}$ irrespective of nanoparticle size and dispersion. Lowering of $M_{s}$ is assigned to a larger magnetically frustrated layer. Those $\mathrm{CS}_{i}$ colloids of MNP coated at lower $\mathrm{pH}_{\mathrm{ads}}$ and displaying larger electrostatic stability present lower $M_{s}$ values. The corresponding $\mathrm{CP}_{i}$ colloids display larger $M_{\mathrm{s}}$ and SAR values consistently with the idea of MNPs having less coating material on their surfaces and being more effectively attracted to the magnet. These observations suggest that citric acid may have an influence on the surface of the MNP, producing larger frustrated layers which on one side improve nanoparticle suspension stability but on the other one lower SAR values. The optimum synthesis condition to accomplish both properties, extremely high stability in suspension at neutral $\mathrm{pH}$ and large SAR values, is obtained by citric acid coating at $\mathrm{pH}_{\mathrm{ads}}=6.25$.

3.4. In Vitro Biocompatibility of CA-Coated MNPs Internalized in A549 Cells. The materials involved in the synthesis procedure (water, CA, and magnetite) are known to display good biocompatibility. However, since interfacial processes may take place when MNPs are dispersed in biological media changing their surface reactivity and consequently their toxicity, ${ }^{34}$ we have tested the viability of our nanoparticles in A549 cells. Figure 14 shows the cytometric analysis of double-labeling for Annexin V and PI assay for the A549 cell without MNP exposure and internalized with three distinct $\mathrm{CS}_{4}$ colloid concentrations. Viable cells are negative for both labelers (DL quadrant); apoptotic cells are PI negative and annexinV positive (DR quadrant); while necrotic cells are positive for both (UR quadrant). After internalization with $\mathrm{CS}_{4}$ colloids at concentrations of 34,67 , and $135 \mu \mathrm{g}_{\mathrm{Fe} 3 \mathrm{O} 4} / \mathrm{mL}$, the percentages of viable cells are $94.7 \%, 95.1 \%$, and $81.3 \%$, respectively. Only for the highest concentration, viability decrease compared with $84.7 \%$ of control cells is observed. The fact that the control culture shows a lower viability than the lower concentrated cultures may be due to an overgrown control culture. It is concluded that $12 \mathrm{~h}$ exposure to CA-coated $\mathrm{Fe}_{3} \mathrm{O}_{4}$ nanoparticles at low concentration is not altering A549 cell viability. Then, citric acid coated magnetite aqueous suspensions at neutral $\mathrm{pH}$ were internalized without causing cell toxicity.

\section{CONCLUSIONS}

We have presented a detailed and extensive study of synthesis conditions, magnetic properties, and radiofrequency dissipation efficiency of various biocompatible citric acid coated magnetite aqueous suspensions at neutral $\mathrm{pH}$. The preparation method enables us to control the long-term stability of the suspension varying the citric acid adsorption $\mathrm{pH}$. The largest stability at neutral medium is achieved for the nanoparticles coated at $\mathrm{pH}$ of 4.58 , where citric acid mainly binds to the particle surface by one carboxylate, leaving two free and charged dangling ends. Citric acid influences the surface of the MNP, producing thick magnetically frustrated layers which on one side improve nanoparticle suspension stability but on the other one reduce SAR values. Finally, our results indicate that dipolar interactions between the nanoparticles play a key role in the Néel relaxation mechanism and dissipation efficiency. Magnetic relaxation becomes slower for interacting colloids, and heating efficiency increases. The largest SAR values arise from the more interacting nanoparticles, a case where Néel relaxation times become larger than Brown relaxation times, leading the Brown dissipation mechanism to prevail. It is shown that SAR data cannot be easily reproduced using the magnetic data and the 

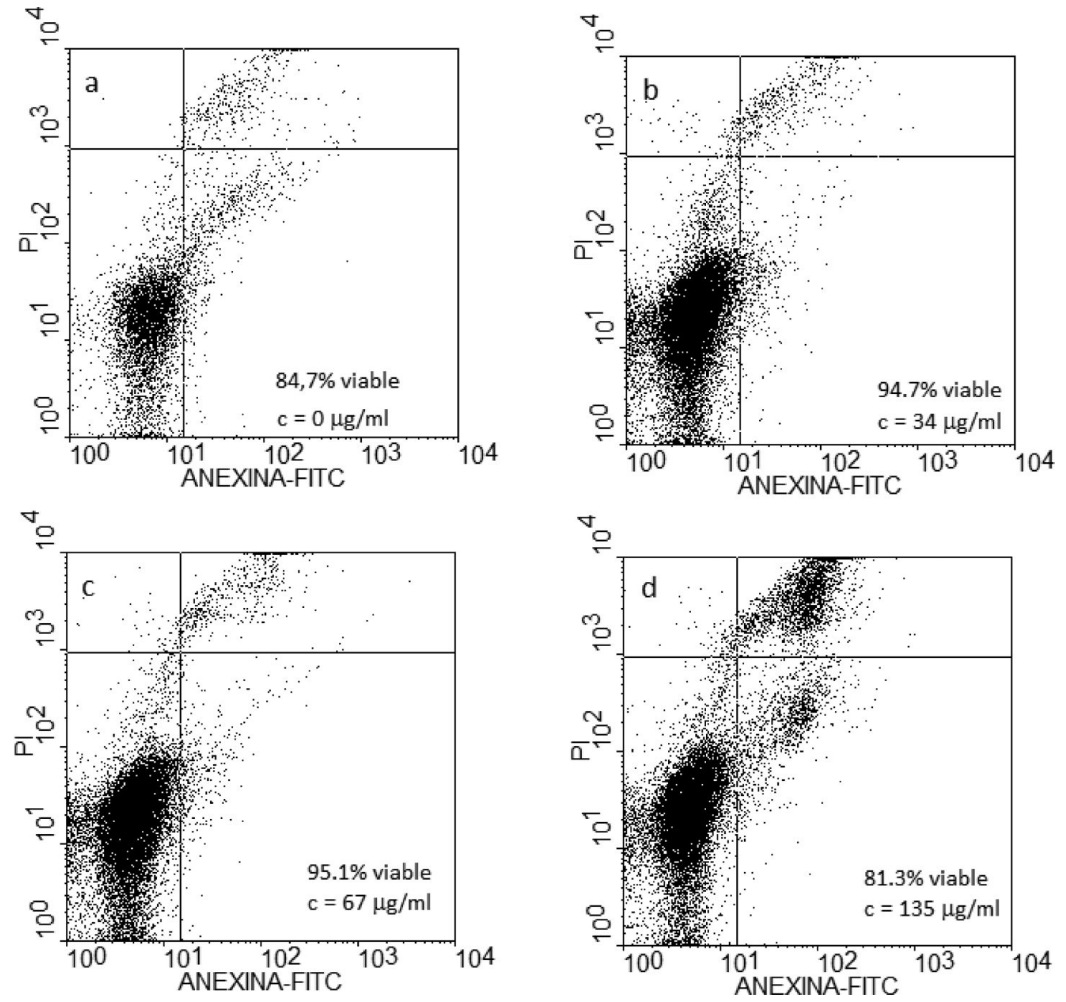

Figure 14. Dot plot obtained from Flow Cytometry data (Annexin/PI staining) of A549 cells. For all plots, Down Left (DL), Down Right (DR), and Up Right (UR) quadrants correspond to viable, apoptotic, and necrotic cells, respectively. Control (a), after $12 \mathrm{~h}$ internalization at $34 \mu \mathrm{g} / \mathrm{mL}$ (b), 67 $\mu \mathrm{g} / \mathrm{mL}$ (c), and $135 \mu \mathrm{g} / \mathrm{mL}$ (d).

most currently used theoretical expressions, due to the presence of dipolar interactions between the nanoparticles. Interaction energy was included in the theoretical expressions through the quantity $\sigma=\left(K_{\text {eff }} V+E_{\text {int }}\right) / k_{\mathrm{B}} T$ and its value inferred from the blocking temperature shift.

\section{ASSOCIATED CONTENT}

\section{S Supporting Information}

Figure S1. This material is available free of charge via the Internet at http://pubs.acs.org.

\section{AUTHOR INFORMATION}

\section{Corresponding Author}

*E-mail: raap@fisica.unlp.edu.ar.

\section{Notes}

The authors declare no competing financial interest.

\section{ACKNOWLEDGMENTS}

This work has been funded by CONICET (PIP 01111), ANPCyT (PICT 00898), and UNLP-X11/556 of Argentina. Zpotential measurements were performed at INQUISUR, UNSCONICET, by V. Lassalle whose help is deeply acknowledged. DC magnetrometry was performed using SQUID equipment of Red de Magnetismo y Materiales Magnéticos (RN3M) and VSM equipment of IFLP-CONICET. We thank FAPESP and C2NANO - Center for Nanoscience and Nanotechnology/ MCT (\# 13872) for the use of TEM equipment. We greatly appreciate the help from R. Dewey and A. Carrea of IIBINTECH (UNSAM) with the flow cytometry analysis and Y. Sosa and R. Goya for help with cell culture experiments, carried out at INIBIOLP - Patología B - CONICET. Marcela B. Fernández van Raap, Patricia Rivas, Pedro Mendoza Zélis,
Gustavo A. Pasquevich, and Francisco H. Sánchez are members of IFLP- CONICET; José L. Alessandrini is a member of Departamento de Física de la UNLP; and M. Elisa de Sousa and Pablo Girardin are fellows of FONCYT and CONICET, Argentina.

\section{ABBREVIATIONS}

AS, ammonia solution; $\mathrm{CA}$, citric acid; $\mathrm{CP}_{i}$, ith pellet colloid; $\mathrm{CS}_{i}$, ith supernatant colloid; DTA, differential thermal analysis; DLS, dynamic light scattering; $d_{\mathrm{H}}$, hydrodynamic diameter; DMEM, Dulbeeco's modified eagle medium; FC, field cooling; IEP, isoelectric point; ISP, interacting superparamagnet; MNP, magnetic nanoparticles; PI, propidium iodide; $\mathrm{p} K_{\mathrm{a}}$ acid dissociation constant; $u$-MNP, uncoated magnetic nanoparticles; rf, radiofrecuency; SAR, specific absorption rate; TEM, transmission electron microscopy; TG, thermogravimetry; XRD, X-ray diffraction; ZFC, zero field cooling; $\sigma_{\mathrm{PI}}$ polydispersity index

\section{REFERENCES}

(1) Rosensweig, R. Ferrohydrodynamics; Dover books on physics; Dover Publications: Mineloa, NY, 1997.

(2) Weinstein, J. S.; Varallyay, C. G.; Dosa, E.; Gahramanov, S.; Hamilton, B.; Rooney, W. D.; Muldoon, L. L.; Neuwelt, E. A. J. Cereb. Blood Flow Metab. 2010, 30 (1), 15-35.

(3) Gupta, A. K.; Gupta, M. Biomaterials 2005, 26, 3995-4021.

(4) Smirnov, P. Methods Mol. Biol. 2009, 512 (II), 333-353.

(5) Dobson, J. Gene Ther. 2006, 13, 283-287.

(6) Chomoucka, J.; Drbohlavova, J.; Huska, D.; Adamb, V.; Kizek, R.; Hubalek, J. Pharmacol. Res. 2010, 62, 144-149.

(7) Hilger, I.; Andrä, W.; Hergt, R.; Hiergeist, R.; Schubert, H.; Kaiser, W. A. Radiology 2001, 218, 570-575. 
(8) Silva, A. C.; Oliveira, T. R.; Mamani, J. B.; Malheiros, S. M. F.; Malavolta, L.; Pavon, L. F.; Sibov, T. T.; Amaro, E., Jr.; Tannús, A.; Vidoto, E. L. G.; et al. Int. J. Nanomed. 2011, 6, 591-603.

(9) Gazeau, F.; Wilhelm, C. Future Med. Chem. 2010, 2 (3), 397408.

(10) Batlle, X.; Pérez, N.; Guardia, P.; Iglesias, O.; Labarta, A.; Bartolomé, F.; García, L. M.; Bartolomé, J.; Roca, A. G.; Morales, M. P.; et al. J. Appl. Phys. 2011, 109, 1-6.

(11) Wei, W.; Quanguo, H.; Changzhong, J. Nanoscale Res. Lett. 2008, 3, 397-415.

(12) Viota, J. L.; Arroyo, F. J.; Delgado, A. V.; Horno, J. J. Colloid Interface Sci. 2010, 344 (1), 144-149.

(13) Kogan, M. J.; Olmedo, M. J. I.; Hosta, L.; Guerrero, A. R.; Cruz, L. J.; Albericio, F. Nanomedicine 2007, 2 (3), 287-306.

(14) Campelj, S.; Makovec, D.; Drofenik, M. J. Phys.: Condens. Matter 2008, 20, 204101/1-204101/5.

(15) Rãcuciu, M. Curr. Appl. Phys. 2009, 9 (5), 1062-1066.

(16) Nigama, S.; Barick, K. C.; Bahadur, D. J. Magn. Magn. Mater 2011, 323, 237-243.

(17) Yallapua, M. M.; Othmanb, S. F.; Curtisb, E. T.; Guptaa, B. K.; Jaggia, M.; Chauhana, S. C. Biomaterials 2011, 32, 1890-1905.

(18) Sahoo, Y.; Goodarzi, A.; Swihart, M. T.; Ohulchansky, T. Y.; Kaur, N.; Furlani, E. P.; Prasad, P. N. J. Phys. Chem. B 2005, 109, $3879-3885$.

(19) Rosensweig, R. E. J. Magn. Magn. Mater. 2002, 252, 370-374.

(20) Massart, R. IEEE Trans. Magn. 1981, MAG-17 (2), 1247-1248.

(21) Lefebure, S.; Dubois, E.; Cabuil, V.; Neveu, S.; Massart, R. J. Mater. Res. 1998, 13 (10), 2975-2981.

(22) Sun, Z.; Su, F.; Forsling, W.; Samskog, P. J. Colloids Interface Sci. 1998, 197 (1), 151-159.

(23) Provencher, S. W. Comput. Phys. Commun. 1982, 27, 213-227.

(24) van Engeland, M.; Nieland, L. J. W.; Ramaekers, F. C.S.; Schutte, B.; Reutelingsperger, C. P. M. Cytometry 1998, 31, 1-9.

(25) Shen, L.; Laibinis, P. E.; Hatton, T. A. Langmuir 1999, 15, 447453.

(26) Panias, D.; Taxiarchou, M.; Paspaliaris, I.; Kontopoulos, A. Hydrometallurgy 1996, 42 (2), 257-265.

(27) Dutta, P.; Manivannan, A.; Seehra, M. S. Phys. Rev. B 2004, 70 (174428), 1-7.

(28) Allia, P.; Coisson, M.; Tiberto, P.; Vinai, F.; Knobel, M.; Novak, M. A.; Nunes, W. C. Phys. Rev. B 2001, 64 (14), 1-12.

(29) Dormann, J. L.; Fiorani, D.; Tronc, E. Magnetic Relaxation in Fine-Particle Systems, Advances in Chemical Physics 98; Wiley: New York, 2007.

(30) Raikher, Y. L.; Shliomis, M. I. The effective field method in the orientational kinetics of magnetic fluids and liquid crystals, Advances in Chemical Physics 87; Wiley: New York, 1994; Chapter 8, p 595.

(31) Gilmore, K.; Idzerda, Y. U.; Klem, M. T.; Allen, M.; Douglas, T.; Young., M. J. Appl. Phys. 2005, 97, 10B301/1-10B301/3.

(32) Fannin, P. C.; Marin, C. N.; Raj, K.; Couper, C.; Barvinschi, P. J. Magn. Magn. Mater 2012, 324, 3443-3447.

(33) Dormann, J. L.; Bessays, L.; Fiorani, D. Solid State Phys. C 1988, 21, 2015-2034.

(34) Casals, E.; Gonzalez, E.; Puntes, V. F. J. Phys. D: Appl. Phys. 2012, 45, 443001/1-443001/15. 


\section{Supporting Information}

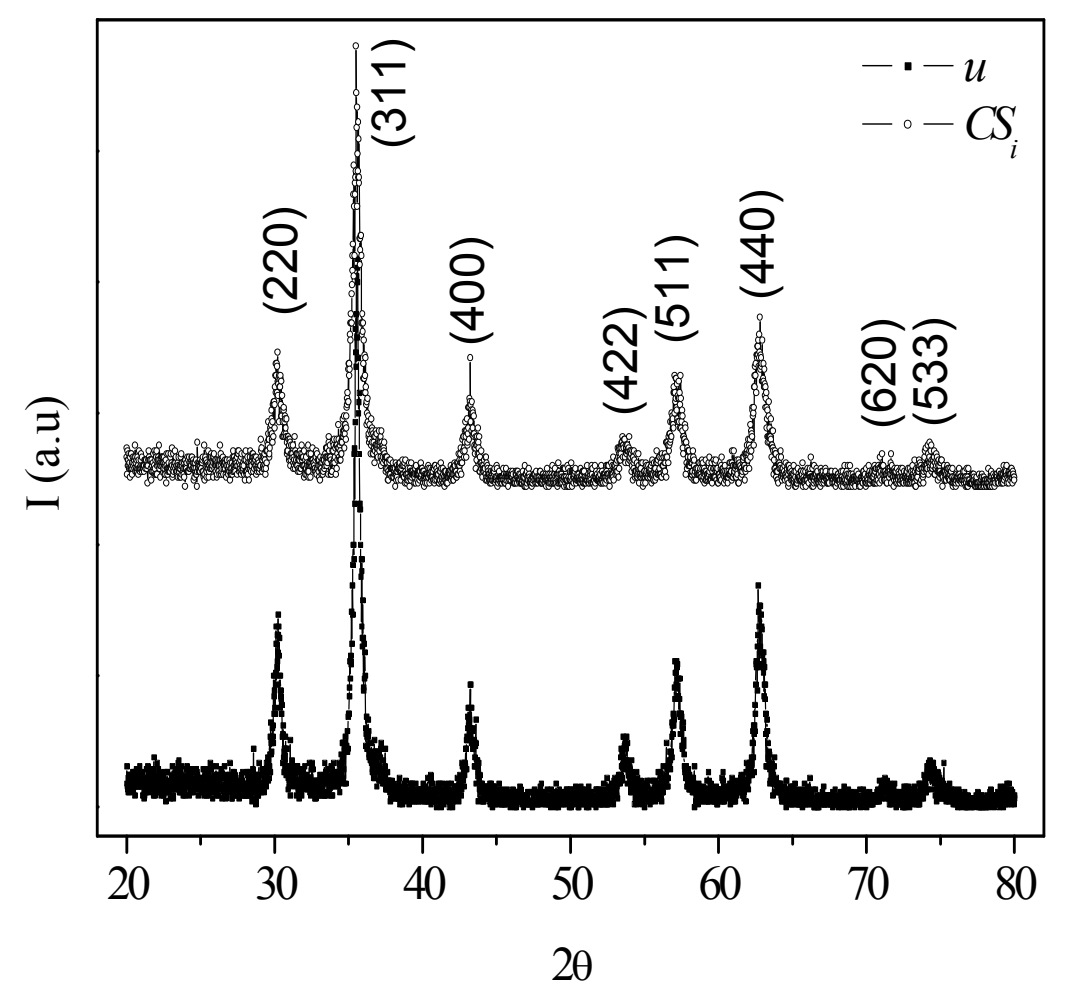

Figure 1: X-ray diffraction pattern of dried powder retrieved from $u$ and $C S_{6}$ colloids with cubic spinel structure (space group $\mathrm{Fd} 3 \mathrm{~m}$ ) indexation.

This information is available free of charge via the Internet at http://pubs.acs.org 